\title{
INTERNACIONALIZAÇÃO NO ENSINO \\ SUPERIOR: (DES) \\ VANTAGENS \\ E DESAFIOS \\ NO CONTEXTO \\ DE UNIVERSIDADE \\ ESTADUAL DO SUL \\ DO BRASIL
}

\section{INTERNACIONALIZACIÓN EN LA EDUCACIÓN SUPERIOR:(DES)VENTAJAS Y DESAFIOS EN EL CONTEXTO DE UNA UNIVERSIDAD ESTATAL DEL SUR DE BRASIL}

\author{
INTERNATIONALIZATION IN HIGHER EDUCATION: THE (DIS) ADVANTAGES AND \\ CHALLENGES IN THE CONTEXT OF A STATE UNIVERSITY IN THE SOUTH OF BRAZIL
}

Édina Aparecida Cabral Bührer* Universidade Estadual do Centro-Oeste

RESUMO: A internacionalização no Ensino Superior está em processo de compreensão da sua significação na voz dos participantes da pesquisa de numa universidade estadual do sul do Brasil. Os participantes informam as (des)vantagens e desafios do processo de internacionalização por meio da oferta de disciplinas em inglês na matriz curricular. A geração de dados ocorreu a partir da análise de entrevistas e questionários com base qualitativa e quantitativa de pesquisa. A interpretação dos dados mostra as vantagens, desvantagens, desafios da internacionalização por meio do Inglês como meio de Instrução (English as a medium of instruction (EMI) e também do domínio da língua na perspectiva do falante não-nativo do inglês. Os dados completos e análise constam no Relatório pós-doutoral realizado na Universidade Federal do Paraná, no período de maio de 2018 a julho de 2019. Neste artigo será apresentada uma síntese do estudo sobre as vantagens, desvantagens e desafios do processo de internacionalização tendo como foco 
principal o EMI.

PALAVRAS-CHAVE: Internacionalização. Educação superior. (Des)vantagens. Desafios.

RESUMEN: La internacionalización en educación superior está en proceso de comprender su importancia a través de la voz de los participantes en la investigación de una universidad estatal del sur de Brasil. Los participantes detallan las (des)ventajas y desafíos en el proceso de internacionalización al ofrecer asignaturas del plan de estudios en inglés. Los datos se obtuvieron a partir del análisis de entrevistas y cuestionarios con base de investigación cualitativa y cuantitativa. A partir de la interpretación de los datos, se observan las ventajas, desventajas y desafíos de la internacionalización a través de English as a Medium of Instruction (EMI), como también el dominio del idioma inglés desde la perspectiva de la construcción de lengua no nativa en inglés. Los datos y el análisis completo se pueden encontrar en el Informe Postdoctoral realizado en la Universidad Federal de Paraná, de mayo del 2018 a julio del 2019. Este artículo presentará una síntesis del estudio sobre las ventajas, desventajas y desafíos del proceso de internacionalización enfocado al EMI.

PALABRAS CLAVE: Internacionalización. Educación superior. (Des)ventajas. Desafíos.

ABSTRACT: The internationalization of Higher Education is in the process of comprehension of its meaning in the research participants' voices in the context of State University in the south of Brazil. The participants present the advantages, disadvantages and challenges of the internationalization process through the medium of English. The data of this study were gathered from interview and questionnaire based on the qualitative and quantitative research. The interpretation of the data shows the advantages, disadvantages and challenges of the internationalization through English as a medium of instruction (EMI) and the knowledge of the English language from the perspective of nonnative speaker of English. The complete results from this study can be found in the final report of a postdoctoral research conducted from May 2018 to July 2019 at Federal University of Paraná, Curitiba, Brazil. This paper will present a synthesis about the advantages, disadvantages and challenges of the internationalization focused on EMI as a process.

KEYWORDS: Internationalization. Higher education. (Dis)advantages. Challenges.

\section{INTRODUÇÃO}

A internacionalização do Ensino Superior, nesta discussão, compreende um processo de integração intercultural, internacional ou de dimensão global em razão, função ou ação de distribuição de educação superior (KNIGHT ${ }^{1}, 2003$, p. 2). Além disso, envolve o conceito de "change-tailored"(KNIGHT, 2012, p. 3), que consiste numa mudança por meio da internacionalização, considerando os interesses e necessidades de cada Instituição de Ensino Superior (IES). Saliento, que mudança está relacionada a ideia de sutura, isto é, algo que vai sendo alinhavado pelas ações/práticas e pelos desejos ${ }^{2}$ de quem se propõe ao processo de transformação por meio da internacionalização do Ensino Superior (e também por quem é conduzido a ela).

Promover a internacionalização do Ensino Superior pode envolver as instituições por diferentes perspectivas, como poderemos observar a seguir, porém, ao relacioná-la ao uso do Inglês, há um envolvimento linguístico direto dos professores. Esse envolvimento ocorre, principalmente, em relação ao uso do EMI, ou seja, "[...] o uso da língua inglesa para ensinar disciplinas acadêmicas (outras que não o Inglês) em países e jurisdições onde a primeira língua (L1) da maioria da população não é o Inglês" ${ }^{3}$ (DEARDEN; MACARO, 2016, p. 456, tradução nossa), pois há professores que não se sentem confortáveis em ensinar usando o inglês e precisam

\footnotetext{
1 "Internationalization at the national, sector, and institutional levels is defined as the process of integrating an international, intercultural, or global dimension into the purpose, functions or delivery of postsecondary education" (KNIGHT, 2003, p. 2).

${ }^{2}$ A ideia de desejo referida faz alusão a Motha e Lin (2014 apud PENNYCOOK, 2017, p. 33) que apontam que no centro de cada aprendizagem da língua inglesa há o desejo pela língua, pelas identidades representadas por um determinado sotaque ou variedade, pelo capital, pelo poder e por imagens associadas ao Inglês que se supõe estarem por trás das portas abertas pela língua inglesa.
}

3 "[...] the use of the English language to teach academic subjects (other than English itself) in countries or jurisdictions where the first language (L1) of the majority of the population is not English" (DEARDEN; MACARO, 2016, p. 456). 
preparar-se para fazê-lo (LAWRENCE et al., 2017). Essa relação, no entanto, será observada neste artigo pelo aspecto geral, ou seja, pela análise da percepção dos professores que responderam ao questionário quanto ao uso do EMI no processo de internacionalização.

Internacionalização do Ensino Superior, portanto, não é algo que acontece rapidamente, tampouco com previsão de finalização, ou seja, é um processo contínuo. Nesse sentido, há um constante repensar de o porquê internacionalizar considerando as possibilidades de cada IES como, por exemplo, o mundo multilíngue que nos rodeia, pois, na relação entre países, principalmente, na integração cultural ou de dimensão global, o papel das diferentes línguas é fundamental para possibilitar uma educação para a cidadania global (CLIFFORD, 2018).

Há, desse modo, diferentes motivos que são considerados por uma IES para internacionalizar, dentre elas destaco a estratégia para atrair estudantes de outros países, a preparação para a mobilidade, o mercado de trabalho globalizado e elevar o perfil classificatório da Universidade (WÄCHTER; MAIWORM, 2014). Além disso, é importante analisar também motivações que requerem um olhar mais crítico em termos de uso do EMI em disciplinas curriculares, isto é, os baixos níveis de competência linguística em língua inglesa e a ameaça à língua da casa (DEARDEN; MACARO, 2016; HULTGREN, 2013).

No processo de internacionalização das universidades, independentemente do nível de ensino, graduação ou pós-graduação, o uso EMI, como parte do processo, suscita um preparo cuidadoso, pois “[...] mudar de um idioma para outro leva tempo, requer paciência e esforço concentrados de todos os envolvidos. Aprender um conteúdo por intermédio de uma segunda língua é desafiador e uma experiência para todos os envolvidos no processo educacional" (MELLION, 2008, p. 224, tradução nossa) ${ }^{4}$.

Reforço, portanto, que os motivos precisam ser cuidadosamente pensados na proposição de implantação do EMI como aliado (ou não) do processo de internacionalização. Sendo assim, na sequência deste artigo, apresentarei a metodologia da pesquisa e os resultados da análise acerca do entendimento dos professores e da administração da IES sobre a internacionalização do Ensino Superior por meio do uso do EMI a partir das (des)vantagens apontadas pelos professores da IES para a utilização do EMI.

\section{METODOLOGIA DA PESQUISA}

Em termos metodológicos, a pesquisa de base qualitativa etnográfica dividiu-se em três etapas: 1) Mapeamento dos estudos sobre internacionalização do ensino superior e EMI; 2) Escolha da Instituição de Ensino Superior (IES) para a execução da pesquisa, mapeamento dos documentos oficiais que informam sobre o processo de internacionalização da IES (resultado já apresentado nos ANAIS eletrônicos do II JILAC), acesso e encaminhamento do questionário semiestruturado via e-mail aos docentes da IES. Nesta fase também houve a entrevista (semiestruturada) com treze membros da administração, coleta e análise de 62 questionários dos docentes (áreas da Saúde, Agrárias, Humanas, Sociais e Ambientais) e da entrevista. Na última etapa foi realizada a análise interpretativa dos documentos, questionários e entrevistas. Os dados das entrevistas não estão contemplados nas porcentagens dos questionários, mas a análise das respostas das entrevistas converge para os resultados observados a partir do questionário.

Conforme mencionado anteriormente, a internacionalização do Ensino Superior, compreendida como processo que envolve o EMI, ocorre de formas diferentes, haja vista que cada IES possui características próprias e dependem tanto de fatores estruturais, para dar suporte ao processo, quanto de professores e alunos com competência linguística. No caso desta pesquisa, a análise da conexão entre internacionalização do Ensino Superior e EMI deu-se a partir da coleta de dados via questionário com 22 perguntas que foram respondidas por 62 professores participantes e um roteiro de perguntas para treze entrevistados (dois representantes de Relações Internacionais, cinco diretores de Setor, dois coordenadores de Curso, um professor de EMI, único em atividade piloto, um diretor de Campus, um coordenador de pós-graduação e um representante de Cursos de Línguas Estrangeiras) e também

4 "Changing from one tongue to another takes time and patience and it requires the concerted efforts of all those involved. This is where the second $\mathrm{C}$ comes in, that of commitment. In the end much more can be gained than just expertise and knowledge. Learning by assimilating content through a second language is a challenging and worthwhile experience for all those involved in the educational process." (MELLION, 2008, p. 224). 
considerando os objetivos de curto prazo propostos num documento oficial da universidade pesquisada. Neste artigo serão analisadas apenas as respostas e justificativas de três perguntas do total de vinte e duas. São elas:

1. Considerando que o inglês tem sido usado como meio de instrução nas disciplinas oferecidas em Universidades nacionais e internacionais, como parte do processo de internacionalização do Ensino Superior, que VANTAGENS você apontaria no uso do Inglês como meio de instrução na Universidade onde trabalha?

2. Considerando que o inglês tem sido usado como o idioma de instrução nas disciplinas oferecidas em Universidades nacionais e internacionais, como parte do processo de internacionalização do Ensino Superior, que DESVANTAGENS você apontaria no uso do Inglês como meio de instrução na Universidade onde trabalha?

3. Qual dos desafios abaixo você apontaria como o maior a ser enfrentado, na Universidade onde trabalha, com a aplicação do uso do Inglês como meio de instrução? Desafio relacionado ao uso do inglês pelo professor; Desafio relacionado ao domínio metodológico e uso do inglês pelo professor; Desafio relacionado à compreensão do inglês pelos alunos; Desafio relacionado ao contato intercultural entre alunos e alunos/professor; Desafio relacionado às diferenças identitárias; Desafio de uso do inglês por professores não-nativos; Desafio relacionado às diferenças socioeconômicas dos alunos. Outro: Justifique a resposta.

No caso das vantagens e desvantagens foi dada uma lista ${ }^{5}$ de opções no questionário e mais uma possibilidade aberta, ou seja, além das opções dadas ainda poderiam ser apontadas outras. Assim, foram listadas sete opções de vantagens do uso de EMI e onze opções de desvantagens ${ }^{6}$. As opções foram criadas a partir de interpretações de artigos especializados que discutem o EMI e temas que deveriam ser pesquisados (WÄTCHER; MAIWORM, 2014; DEARDEN, 2014; MACARO et al., 2018). As respostas dos 62 professores foram analisadas considerando o número de vezes que uma mesma opção foi apontada por diferentes docentes.

A partir da análise buscou-se estabelecer relações gerais, ou seja, entre o Tratado de Bolonha ${ }^{7}$ e os motivos da internacionalização, mencionados anteriormente. A relação deu-se a partir das alternativas escolhidas pelos participantes quando se referiram à implantação do EMI em disciplinas do currículo, pois, nesse aspecto, parti do princípio de que "[...] no processo de Bologna, internacionalização, significa educação superior por meio do inglês” (PHILLIPSON, 2009 apud KIRKPATRICK, 2014, p. 4, tradução nossa). Ainda, apesar de parecer um tanto quanto exagerado, não se pode negar o aumento significativo de programas de EMI (KIRKPATRICK, 2014), pois o processo de internacionalização, via uso do inglês, assume um tom mais complexo, principalmente, ao se privilegiar "English Only" e o "native speaker model" (KIRKPATRICK, 2014). A complexidade, neste caso, dáse ao privilegiar um único tipo de Inglês conectado às bases tradicionais do idioma como primeira língua diretamente relacionado aos países do Círculo Interno (CRYSTAL,1997; McKAY, 2002).

Ao tecer essa rede de nós, que envolve o processo de internacionalização via EMI ou como principal tópico, sigo para a descrição e análise dos aspectos delimitados na introdução.

\section{DESCRIÇÃO E ANÁLISE}

\subsection{EMI: OPORTUNIDADE E GLOBALIZAÇÃO}

Iniciando pelas vantagens, a implantação do EMI em disciplinas, como parte do processo de internacionalização, traria, de acordo

\footnotetext{
${ }^{5}$ (Ex.: 1- Oportunidade para o ingresso dos estudantes do Ensino Superior no mundo acadêmico e profissional global; 2- Melhoria da capacidade de uso do inglês pelos alunos; 3- Acesso da Universidade no mercado competitivo global; 4- Preparar o aluno para a diversidade linguística; 5- Promover a Universidade em escala global; 6-Atrair estudantes estrangeiros para a Universidade; 7- Criar e fortalecer as relações entre países; 8- Abrir as portas para a globalização).

${ }^{6}$ (1- Elitização do Ensino Superior; 2- Dificuldade de Interação entre alunos e professor em sala de aula; 3- Intensificação das diferenças culturais e dos níveis de conhecimento de inglês; 4- Enfraquecimento da identidade linguística local; 5- Favorecimento do inglês em detrimento de outras línguas: 6- Desrespeito à cultura local: 7- Favorecimento de um pequeno número de alunos; 8- Pouco ou nenhum suporte pedagógico para o aluno e professor na aprendizagem do inglês; 9- Poucos professores com conhecimento de inglês o suficiente para conduzir a aula; 10- Ausência de recursos financeiros para dar suporte ao uso do inglês como meio de instrução; 11- Ausência de um ambiente interativo em sala de aula entre alunos e professor por meio do inglês).
}

${ }^{7} \mathrm{O}$ Tratado de Bolonha pode ser entendido aqui como um modelo de Ensino Superior Europeu, originado na União Europeia por meio da promoção da movimentação de pessoas entre instituições, instigando a autonomia e a aprendizagem tendo como alvo o desenvolvimento de capacidades diretamente relacionadas ao mercado de trabalho, seja nacionalmente ou internacionalmente (MACEDO, 2017). 
com os pesquisados, oportunidades para o ingresso dos estudantes do Ensino Superior ao mundo acadêmico e profissional global (alternativa 1). A alternativa teve o maior número de ocorrências (35), ou seja, dos 62 participantes, 35 apontaram a alternativa de número 1 como uma vantagem da internacionalização via EMI. Além dessa, houve outras ocorrências, tais como:
2) Promover a Universidade em escala global (28 ocorrências);
3) Melhoria da capacidade de uso do inglês pelos alunos (27 ocorrências)
4) Atrair estudantes estrangeiros para a Universidade (26 ocorrências);
5) Preparar o aluno para a diversidade linguística (26 ocorrências).

De acordo com a análise interpretativa, há uma relação direta entre internacionalização e EMI porque promoverá oportunidades e melhorias na e para a Universidade, preparará alunos e professores para o mundo globalizado e atuará como um atrativo para o mercado, mediada pela língua inglesa globalizada. As relações vantajosas apontadas pelos participantes, podem, por exemplo, ser ainda ponderadas sob a perspectiva da identidade institucional, ou seja, como as instituições querem ser vistas mundo afora ou como elas são percebidas (ranqueadas, categorizadas) pelo resto do mundo (BRADFORD, 2016; MARTINEZ, 2016, p. 201). Segundo a análise, a língua inglesa poderia dar acesso à universidade em estudo pela construção de uma imagem de IES que produz artigos, promove intercâmbios em língua inglesa e, portanto, faz parte do mundo globalizado. Porém, neste mundo globalizado, as universidades são pensadas como mercado e alimentadas pelo recrutamento de estudantes internacionais à medida que competem para serem aceitos e trazem consigo as mensalidades (SOREN, 2013, p. 2).

Uma visão de universidade para o mercado e recrutamento vai ao encontro das justificativas dadas pelos participantes sobre o uso do EMI quanto às vantagens, pois percebe-se uma relação com a posição da IES, do aluno e também dos docentes, no mercado de trabalho globalizado numa perspectiva de possibilitar um status global de IES. Observe algumas das justificativas:

1- "Uma Universidade só estará no processo de internacionalização se o inglês estiver cada vez mais presente nas suas disciplinas". 2-“A utilização do EMI favorece a mobilidade docente e discente e a circulação de conhecimentos em inglês".

3-"O inglês é uma língua global. Sendo assim, o aperfeiçoamento do idioma, via a participação do aluno em uma disciplina ministrada em inglês, promoveria maior inserção do aluno no mercado profissional global”.

4-“O Inglês é a língua universal, usada para comunicação em qualquer local do mundo”.

5-“A internacionalização é inevitável. O inglês é a língua da internacionalização, como o latim o foi na idade média até os primeiros séculos da modernidade".

6-“Em países desenvolvidos, e mesmo outros de terceiro mundo, a maioria das pessoas é fluente em inglês”.

As vantagens relacionadas ao uso do EMI, num contexto de internacionalização na universidade pesquisada, podem apontar em direção à mencionada por Dearden e Macaro (2016), isto é, de que EMI parece ser entendido por professores como um passaporte para o mundo global, uma chave para o sucesso, uma oportunidade para expandir os horizontes dos alunos.

A partir da categorização de Bradford (2016) sobre os desafios institucionais, abordada por Martinez (2016), como relacionada à identidade institucional, podemos inferir também que, no caso das vantagens apontadas pelos participantes, há uma relação entre EMI e preparo para a competitividade no mundo do trabalho em escala global e, portanto, a construção de uma identidade institucional mais competitiva.

Uma questão que parece pendente, apesar de aparecer em 26 ocorrências, nessa perspectiva de vantagens, é a promoção da diversidade linguística ou de uma integração intercultural efetiva. Ao mencionar a diversidade linguística como algo pendente, entendo que este tipo de discussão necessita de uma análise mais profunda da concepção de EMI, como apontado no início desta discussão, e ultrapassar a relação estabelecida com a língua padrão ${ }^{8}$, pois EMI parece apresentar uma estreita relação com native-

\footnotetext{
${ }^{8}$ Língua padrão é aquela variedade que tem o mais alto status em uma sociedade geralmente baseada na escrita e na fala de falantes educados de uma língua, usada na mídia e literatura, descrita em dicionários e gramáticas ensinada nas escolas e para não-nativos quando eles aprendem a língua como língua estrangeira. (McKay,
} 
like, principalmente na perspectiva de "off shore universities" (JENKINS, 2011, p. 933, ênfase da autora). Nesse caso, a pendência ocorre justamente pelo tipo e propósito do uso do inglês.

As vantagens apontadas pelos participantes, portanto, em maior ou menor grau, informam que o EMI pode criar expectativas de impactar positivamente tanto na visibilidade da IES quanto nas condições de competitividade para os estudantes, em uma relação que não é tão direta quanto parece. Quanto ao possível posicionamento da Universidade estudada num processo de internacionalização via EMI, não me privo da reflexão sobre o tipo de Universidade que se busca para que a diversidade linguística possa ter alguma possibilidade de ocorrer ou para que a internacionalização do Ensino Superior supere a perspectiva de responder ao mercado. Assim, a reflexão aqui ocorre considerando principalmente: "Que tipo de Universidade para que tipo de sociedade? Que Universidade e sociedade esperam uma da outra? Como a Universidade espera adequar-se dentro de uma instituição democrática e social? Em que extensão e como Universidade, governo e sociedade pretendem influenciar umas às outras? Qual é a extensão e direção da mudança?" ${ }^{9}$ (OLSEN, 2005, p. 3 tradução nossa).

Na relação entre o tipo de Universidade que se quer e que a sociedade precisa, é importante analisar também como o EMI contribui ou não para a cidadania global, isto é, as pessoas diretamente implicadas com o processo de internacionalização precisam pensar na responsabilidade social (CLIFFORD, 2018). É importante pensar ainda, que se EMI se comporta como elemento de "marketization" (COLEMAN, 2006), as vantagens podem ter outra conotação quando se pensa que a "[...] combinação de taxas altas individuais, aumento de mobilidade estudantil e excesso de oferta diante da procura têm acentuado o caráter de mercado do Ensino Superior: os estudantes tornaram-se clientes. Universidades não são mais instituições, mas produtos (COLEMAN, 2006, p. 3) ${ }^{10}$. Nesse aspecto, a possibilidade de internacionalização do currículo via EMI, prevista em documentos da Universidade estudada pode ser pensada pela tentativa de projetar a IES num patamar de discussão que a desafie a pensar nas necessidades de todos os estudantes, indo além da ideia de que internacionalizar o currículo pode também significar dar suporte para que os alunos se adaptem ao estilo de educação "ocidental" (CLIFFORD, 2018, p. 24).

A perspectiva da identidade institucional que se interpreta das respostas dos participantes pode ser relacionada ao reconhecimento da instituição no contexto global ligado ao movimento de pessoas e intimamente conectado ao uso de um inglês globalizado, mas que também posiciona o professor num contexto mais amplo. Nesse contexto de identidades institucional e pessoal, questões como quem é o professor dentro dessa Universidade e que posição ocupa podem exercer certo poder em relação ao contexto global na perspectiva de ser reconhecido como "um certo tipo de pessoa" dentro de um determinado contexto (GEE, 2000).

Essa perspectiva de identidade institucional, no entanto, pode esbarrar nas desvantagens de uso do EMI apontada na pesquisa, pois, segundo 54\% deles, há poucos professores com conhecimento de inglês o suficiente para conduzir uma aula usando EMI nos moldes esperados. Essa desvantagem está relacionada ao nosso próximo assunto, as desvantagens e desafios de uma implantação do EMI no currículo.

As desvantagens e desafios conduziram a discussão para o conhecimento da língua inglesa e, neste caso, seus correlatos, domínio, fluência e proficiência. O que se percebeu na pesquisa, e será apontado na sequência, é uma ideia de conhecimento de língua que se confunde (ou se funde) com seus correlatos. Nesse sentido, a relação foi estabelecida com o modelo de falante nativo, pois as expressões fluência e proficiência têm sido tradicionalmente testadas seguindo um modelo padrão e servindo como certificação de domínio da língua inglesa.

2002, p. 51, tradução nossa).

9 "The current dynamics raise questions about the University's long-term pact with society: What kind of University for what kind of society? What do the University and society expect from each other? How is the University assumed to fit into a democratic polity and society? To what extent and how, are the University, government and society supposed to influence each other? What is the extent and direction of change?" (OLSEN, 2005, p. 3).

10 "The combination of higher individual fees, greater student mobility, and excess of supply over demand has accentuated the market character of HE: the student has become the customer. Universities are no longer institutions but brands." (COLEMAN, 2006, p. 3). 


\subsection{EMI: DESVANTAGENS E DESAFIOS}

Discutir acerca do domínio da língua inglesa, mesmo no contexto de EMI, pode gerar inúmeras indagações, por isso esclareço que não foi solicitado aos participantes, que responderam ao questionário tampouco na entrevista, qualquer definição de domínio, proficiência, fluência ou conhecimento de língua. Não estava no objetivo de a pesquisa definir qualquer um deles, mas sim entender as desvantagens que a implantação do EMI poderia trazer no contexto da IES. Os participantes usaram as expressões, mencionadas anteriormente, como uma forma de mostrar o lugar do EMI no contexto da IES em termos de níveis de uso da língua.

De acordo com os eles, portanto, as desvantagens do uso do EMI em disciplinas, como parte do processo de internacionalização, estariam centradas, com um número maior de ocorrências, em:

\begin{tabular}{c|c} 
Favorecimento de um pequeno número de alunos; & $15(24 \%)$ \\
\hline Pouco ou nenhum suporte pedagógico para o aluno e professor na aprendizagem do inglês; & 14 \\
\hline Poucos professores com conhecimento de inglês o suficiente para conduzir a aula & $34(54 \%)$ \\
\hline Ausência de recursos financeiros para dar suporte ao uso do inglês como meio de instrução; & $23(37 \%)$ \\
\hline Ausência de um ambiente interativo em sala de aula entre alunos e professor por meio do inglês. & 12
\end{tabular}

Tabela 1: Desvantagens no uso inglês meio de instrução

Fonte: Bührer (2019)

Como se pode observar, um número considerável de ocorrências (dentro dos parâmetros da pesquisa) deu-se em relação à preocupação de que há poucos professores com conhecimento de inglês o suficiente para conduzir uma aula usando o EMI. A interpretação do que significa conhecimento do idioma fica subjetivo se analisada pela perspectiva quantitativa, mas entende-se que, segundo eles, o conhecimento é insuficiente para a prática do EMI.

A análise das justificativas relacionadas aos desafios do EMI tornou possível um entendimento mais claro acerca das preocupações dos participantes com o conhecimento do idioma. Assim, as reiterações das palavras domínio, proficiência, fluência e conhecimento da língua foram observadas quando perguntei aos participantes qual seria o maior desafio para a implantação do EMI na universidade (haja vista que o EMI não é ainda uma prática institucionalizada na IES estudada, mas faz parte de plano institucional). Entre os grandes desafios apontados pelos participantes, dois deles tiveram mais ocorrências:

a) Desafio relacionado à compreensão do inglês pelos alunos com 29 (vinte e nove) ocorrências;

b) Desafio relacionado ao uso do inglês pelo professor com 10 (dez) ocorrências;

c) Desafio de uso do inglês por professores não-nativos com 8 (oito) ocorrências.

Algumas das justificativas foram:

Penso que o grande empecilho é a falta de fluência dos professores. (Participante 6A).

Embora essencial, o domínio do idioma inglês ainda é menosprezado e apresenta muita resistência pelos docentes de forma geral. (Participante 7A).

Poucos professores e alunos têm domínio na língua. (Participante 8A).

Nossos alunos em grande maioria não compreendem sua língua mãe e muito menos o Inglês. (Participante 9A).

Pouco conhecimento do idioma pelos alunos e pouco domínio do idioma por vários professores. (Participante 10A).

Pouco alunos têm proficiência necessária para acompanhar uma aula em Inglês. (Participante 11 A). 
A maioria dos professores não são fluentes. (Participante 12A).

Poucos alunos dominam tal idioma tanto na graduação quanto na Pós [graduação]. (Participante 13A).

Os alunos brasileiros, em sua maioria, não possuem proficiência para o entendimento do Inglês em geral e, em especial, os termos técnicos da área, o que prejudicaria e dificultaria o aprendizado. (Participante 15A).

A origem dos alunos, provenientes de escolas públicas onde o nível de conhecimento e de formação é baixíssimo. (Participante 16A).

O brasileiro possui pouco conhecimento em Inglês (...). (Participante 17A).

Muitos professores, mesmo que pós-graduandos, não têm fluência na língua, ao contrário dos alunos. (Participante 18A).

Embora o conhecimento da maioria dos professores seja insuficiente para conduzir uma aula em Inglês, esse obstáculo poderia ser mais facilmente contornado do que a compreensão dos alunos em Inglês, pois a grande maioria possui apenas um conhecimento muito básico. (Participante 19A).

Para que a disciplina seja efetiva deve haver domínio da língua por parte do professor e dos alunos. (Participante 20A).

Pouco conhecimento sobre a língua Inglesa e distância com a língua materna. (Participante 21A).

Falta domínio básico da língua para os alunos em conversação e compreensão. (Participante 22A).

Muitos alunos não possuem proficiência em língua inglesa. (Participante 23A).

Acredito que tanto professor como alunos precisam antes conhecer a língua inglesa para então trabalhar uma disciplina nessa perspectiva. (Participante 24A).

Devido à necessidade de aprofundar o entendimento dos participantes, que escolheram o desafio relacionado ao uso do inglês por professores não-nativos (oito ocorrências), decidi verificar quais as justificativas dadas para tal escolha e, na sequência, estabelecer possíveis relações com ideia de domínio da língua e estudos acerca do construto falante nativo/não-nativo de inglês. Vale ressaltar que, dos oito participantes, três deles não justificaram. Assim, temos:

Embora essencial, o domínio do idioma inglês ainda é menosprezado e apresenta muita resistência pelos docentes de forma geral (Participante 1A)

Nossa Universidade é pública e recebe alunos das mais diversas condições socioeconômicas o que dificultaria essa prática pois muitos deles não têm a oportunidade de estudar inglês para esse tipo de desafio, mas acredito que um início de processo também ajudaria os professores e esses alunos a superarem juntos. (Participante 2A)

Muitos professores, mesmo que pós-graduados, não tem fluência na língua, ao contrário dos alunos (Participante 3A)

Poucos professores teriam como realmente trabalhar em inglês. (Participante 4A)

Se o professor não tem, minimamente, o conhecimento da língua inglesa e boa proficiência, a relação ensino-aprendizagem deverá ficar muito comprometida (Participante 5).

A partir da relação estabelecida pelos participantes com domínio, proficiência e fluência, entendo que adentramos num campo interpretativo que se conecta com "native speaker model" (KIRKPATRICK, 2014) e, portanto, numa perspectiva de uso do inglês ligado ao Círculo Interno, ou seja, refere às bases tradicionais do Inglês, nos países onde é usado como primeira língua. Isso inclui países como Estados Unidos, Reino Unido, Irlanda, Canadá, Austrália e Nova Zelândia, conforme Crystal (1997)). Dessas relações e conexões, outras relações acontecem como por exemplo entre falante não-nativo com EMI, observado pelos dados da pesquisa, que, por sua vez, pode conduzir ao campo de discussões sobre falante nativo e falante não-nativo de língua inglesa (FN/FNN), já registrado em estudos anteriores, cujo significado do construto é questionado.

Peter Medgyes $(1992,1994)$, por exemplo, foi pioneiro ao tematizar a relação numa perspectiva crítica e contestadora. Segundo o autor, os FNN podem ser professores de sucesso, mas as rotas são diferentes do FN. O construto FN/FNN é questionado em vários níveis, entre eles, o entendimento de falante nativo, por exemplo: 
Quem é o falante nativo de Inglês? O Bretão é. O Húngaro não é. Um australiano é. Um francês nacional não é. Até agora tudo bem. Mas o que dizer de um Indiano para quem o Inglês foi a língua de instrução na escola e língua da comunicação profissional desde sempre? Ele não se encaixa como nativo nem como não-nativo. De fato, países onde o Inglês é uma segunda língua quebra a hegemonia da divisão nativo/não-nativo. O problema é que a divisão nem sempre se aplica aos chamados falantes dos países de língua inglesa também.

Vamos usar o exemplo de Juan, idade 9, que vive nos Estados Unidos desde os 5 anos. O pai dele é um imigrante mexicano. A mãe veio da Noruega. Ambos falam com Juan na língua materna da mãe. Qual é a língua nativa de Juan, Inglês, Espanhol ou Norueguês? Todas as três? Nenhuma delas? (MEDGYES, 1992, p. 340, tradução nossa 11)

A partir das justificativas dos participantes, definir conhecimento e domínio de língua com EMI e relacioná-las ao construto FN/FNN parece conduzir para novas reflexões, pois apesar dos FNN estarem em maioria no campo de ensino de inglês, há preferência de aprendizes pelos FN (ALGHOFAILI; ELYAS, 2017).

Medgyes (2017, p. 11) aborda as tentativas de definição do FN e alerta para os critérios inconsistentes e difusos, tais como:

Como podemos definir a língua nativa em relação à língua mãe, primeira língua, língua 1, língua dominante, língua de casa. E de frases como segunda língua e língua estrangeira? A que ponto esses rótulos são intercambiáveis? Confusão parecida se dá com conceitos como comando do Inglês, conhecimento do Inglês, competência em inglês ou proficiência em Inglês. O termo proficiência native-like em inglês revela claramente a insegurança dos pesquisadores. Como professores de Língua, todos nós sabemos que há muitos meios de se medir a proficiência linguística. Apesar disso, não há um padrão de medição na qual nativos podem servir para distinguir dos não-nativos. Num teste para medir a "natividade" de um indivíduo começamos, digamos assim, por cem itens? (MEDGYES, 2017, p. 12)

A fim de aliar domínio, fluência, proficiência, FN/FNN com o EMI é importante estabelecer de que forma se entende o uso do EMI no contexto do Ensino Superior para poder relacionar (ou não) ao professor FNN. Assim, mais pesquisas precisam ser realizadas para que se possa ter um entendimento mais profundo das relações entre EMI e FNN, porque não se pode afirmar que o Inglês do EMI está diretamente relacionado ao Inglês vinculado aos países do Círculo Interno, portanto, ao FN. Apesar disso, como já dito anteriormente, há indícios de que as políticas do "English-only ${ }^{12 "}$ podem estar, na maioria das vezes, relacionadas ao inglês nativo e não ao inglês como língua franca, o que nos faz pensar novamente no processo de dominação da língua inglesa padrão porque o I do Inglês no contexto do uso Só-Inglês está quase sempre baseada no falante nativo. (KIRKPATRICK, 2014, p.0 9, tradução nossa) $)^{13}$.

Relacionar o EMI ao Inglês como língua franca exige, entre outras coisas, refletir com mais profundidade acerca de uso. Outra questão analisada na pesquisa de pós-doutorado foi a experiência de um dos participantes na condução do EMI em sala de aula como uma experiência piloto. Nela observei uma proximidade maior da perspectiva de EMI com o construto FN/FNN quando o participante se posicionou ora numa perspectiva de uso comunicativo da língua Inglesa, ora como alguém que precisa de um conhecimento maior da língua quando em contato com falantes nativos. Essa reflexão, no entanto, ficará para outro momento. Por ora apresentei os dados de uma parte da pesquisa e saliento que o tema é instigante porque exige que pensemos na proficuidade da internacionalização, ou seja, para que ela serve ou para quem ela serve.

\footnotetext{
11 "Now the question is: who is a native speaker of English? A Briton is. Hungarian is not. An Australian is. A French national is not. So far, so good. But what about an Indian for whom English was the language of school instruction and has been the language of professional communication ever since? Let us take Juan, for example, aged 9, who has been living in the United States for five years. His father is a Mexican immigrant; his mother comes from Norway. They both speak to Juan in their own mother tongue. Which is his native language, English, Spanish, or Norwegian? All three of them? None of them?" (MEDGYES, 1992, p. 340).

${ }^{12}$ Política de usar somente o Inglês em sala de aula. Proponentes do "Somente-Inglês" argumentam que os alunos precisam ouvir e usar o Inglês quanto o mais possível. (KIRKPATRICK, 2014).
}

13 “"E” do English only policy is almost always based on a native speaker" (KIRKPATRICK, 2014, p.0 9). 


\section{CONCLUSÃO}

A partir dos dados apresentados no artigo, entendo que as vantagens de uso do EMI em disciplinas da matriz curricular dos cursos de graduação estão inseridas numa perspectiva de promoção da universidade, melhoria da capacidade do uso do inglês, atração de estudantes estrangeiros e preparação para a diversidade linguística, mas por outro lado, as desvantagens apontam para o número reduzido de professores com conhecimento de inglês o suficiente para conduzir uma aula, a ausência de recursos financeiros para dar suporte ao uso do inglês num contexto de EMI, o favorecimento de um grupo pequeno de alunos, pouco ou nenhum suporte ao uso do inglês como meio de instrução e ausência de um ambiente interativo em sala de aula entre alunos e professor por meio do inglês. Além disso, os participantes apontaram o uso do inglês pelo professor, a compreensão do inglês pelo aluno e o uso do inglês por professores não nativos como desafios que pensam enfrentar na universidade em estudo no processo de implantação do EMI.

As (des)vantagens e desafios, no entanto, foram analisados levando em consideração os motivos que levam uma IES ao processo de internacionalização do Ensino Superior usando o EMI em disciplinas no currículo. As motivações podem diferenciar de uma universidade para outra a partir, certamente, da possibilidade de cada uma em superar os desafios e transformar as desvantagens. No entanto, a análise da pesquisa se faz tendo uma perspectiva crítica de aplicação ou implantação do EMI no sentido de que é preciso, além de compreender a própria identidade institucional, ir além dos muros da própria IES e analisar os interesses da internacionalização do Ensino Superior em relação ao uso da língua inglesa vinculado ao falante nativo, recrutamento de estudantes internacionais (fuga de cérebros) com fins inteiramente vinculados ao mercado de trabalho e ao EMI como "marketization".

\section{REFERÊNCIAS}

ALGHOFAILI, N. M.; ELYAS, T. Decoding the Myths of the Native and Non-native English Speakers Teachers (NESTs \& NNESTs) on Saudi EFL Tertiary Students. English Language Teaching, Canadian Center of Science Education, v. 10, n. 6, p., 2017.

BRADFORD, A. Toward a Typology of Implementation Challenges Facing English-Medium Instruction in Higher Education: Evidence From Japan. Journal of Studies in International Education, Japan, vol. 20, n.3, p. 339-356, n3. 2016.

CABRAL-BÜHRER. E. A. Internacionalização e EMI em uma universidade estadual do sul do Brasil: (des) vantagens e desafios. Curitiba: UFPR, 2019. 80 f. (Relatório de Pós-doutorado).

CLIFFORD, V. Exploring internationalization of the curriculum through the lens of global citizenship. In: LUNA, J. M. F. de. (org.). Internacionalização do Currículo- Educação-Interculturalidade-Cidadania global. 2. ed. Campinas, SP: Pontes Editores, p. 13-31, 2018.

COLEMAN, J. A. English-medium teaching in European Higher Education. Language Teaching, United Kingdom, v. 39, n. 1, p. 114, 2006.

CRYSTAL, D. English as a Global Language. Cambridge: Cambridge University Press, 1997.

DEARDEN, J. English as a medium of instruction - a growing global phenomenon. London: British Council, 2014. Disponível em: Disponível em:https://www.britishcouncil.es/sites/default/files/british council english as a medium of instruction.pdf Acesso em:16 abr 2019

DEARDEN.J; MACARO, E. Higher Education teacher's attitudes towards English medium instruction: A three-country comparison. Studies in second language Learning and teaching. Kalisz, v. 6, n.3, p. 455-486, 2016. Disponível em: https//www.cambridge.org/core Acesso em: 12 jun. 2018. 
GEE, J. Identity as an Analytic Lens for Research in Education. Review of Research in Education, [s.1], v. 25, n.1, p. 99-125, 2000. Disponível em: .https://pdfs.semanticscholar.org/17bc/c796536ea383de959cc3bd97bd886b579754.pdf Acesso em: out. 2012.

HULTGREN, A.K. Lexical borrowing from English into Danish in the sciences: An empirical investigation of domain loss. International Journal of Applied Linguistics, v. 23, n. 2, p. 166-182, 2013.

JENKINS, J. Accommodating (to) in the international university. Journal of Pragmatics, United Kingdom, n.43, p. 926-936, 2011.

KIRKPATRICK, A. “The Language(s) of HE: EMI and/or ELF and/or Multilingualism?” The Asian Journal of Applied Linguistics, v. 1, p. 4-15, 2014.

KNIGHT, J. Five truths about internationalization. International Higher Education, n. 69, p.4-5, fall 2012.

KNIGHT, J. Updating the definition of internationalization. International Higher Education, n. 33, p.2-3, 2003.

LAWRENCE, C; INBAR-LOURIE, O; WEINBERG, L. A Handbook for English-Medium Instruction in Institutions of Higher Education in Israel. English as the Cornerstone of Sustainable Technology and Research. [s.l]: ECOSTAR, 2017. Disponível em: https://tempus-ecostar.iucc.ac.il/wp-content/uploads/2017/05/EMI-BOOK-ATAR.pdf. Acesso em: 6 jun. 2018.

MACARO, E. et al. A systematic review of English medium instruction in higher education. Language Teaching, v.51 n1, p. 36-76, 2018.

MACEDO, B. O Processo de Bolonha: Discursos e dinâmicas da reformulação educacional nas universidades da União Europeia. Algumas reflexões. Debater a Europa. Periódico do CIEDA e do CEIS2, n.16, jan./jun. 2017. Disponível em: http://www.europedirect-aveiro.aeva.eu/debatereuropa/https://doi.org/10.14195/1647-6336 169

MARTINEZ, R. English as a medium of Instruction (EMI) in Brazilian higher education: challenges and opportunities. In: FINARDI, K. R. (org.). English in Brazil: views, policies and programs. Londrina: Eduel, 2016. p. 193-228,

McKAY, S.L. Teaching English as an International Language: Rethinking Goals and Approaches. Oxford: Oxford University Press, 2002.

MEDGYES, P. Native or non-native: Who's worth more? ELT Journal, Oxford, v. 46, n. 4, p., 1992.

MEDGYES, P. The non-native teacher. London: Macmillan Publishers, 1994.

MEDGYES, P. The non-native teacher. Updated edition with new material. Scotland, UK: Swan, 2017.

MELLION, M. The challenge of changing tongues in business university education. In: Realizing Content and Language Integration in Higher Education. Maastricht: Maastricht University. p. 212-227, 2008. Disponível em: https://ru.on.worldcat.org/oclc/1106016186. Acesso em: 3 abr. 2019.

OLSEN. J.P. The institutional dynamics of the (European) University. ARENA, Centre for European Studies. University of Oslo. Working paper, n. 5, 2005. Disponível em: https://www.sv.uio.no/arena/english/research/publications/arena-workingpapers/2001-2010/2005/wp05 15.pdf. Acesso em: 6 jun. 2018.

PENNYCOOK, A. The Cultural Politics of English as an International Language. London: Routledge, 2017.

SOREN. J. K. Teacher Identity in English-Medium Instruction: Teacher Cognitions from a Danish Tertiary Education Context. 2013.

Bührer | Internacionalização no ensino superior: (des)vantagens e desafios no contexto de universidade estadual.. 
PhD. Denmark: University of Copenhagen, 2013. Disponível em: $\underline{\text { https://hal.archives- }}$ ouvertes.fr/search/index?q=Joyce+Kling+Soren Acesso em: 21 mar. 2019.

WÄTCHER, B; MAIWORM, F. English-Taught Programs in European higher education: The state of play in 2014. Disponível em: http://www.aca-secretariat.be/fileadmin/aca docs/images/members/ACA-2015 English Taught 01.pdf Acesso em: 6 jun. 2018.

\section{(ㄷ) (1) $\circledast$}

Recebido em 06/03/2020. Aceito em 13/08/2020. 